Georgian Mathematical Journal

1(1994), No. 2, 151-172

\title{
ON COHOMOTOPY-TYPE FUNCTORS
}

\author{
S. KHAZHOMIA
}

\begin{abstract}
This article deals with Chogoshvili cohomotopy functors which are defined by extending a cohomology functor given on some special auxiliary subcategories of the category of topological spaces. The question of choosing these subcategories is discussed. In particular, it is shown that in the singular case to define absolute groups it is sufficient that auxiliary subcategories should have as objects only spheres $S^{n}$, Moore spaces $P^{n}(t)=S^{n-1} \cup_{t} e^{n}$, and one-point unions of these spaces.
\end{abstract}

In $[2,3]$ for any cohomology theory $H=\left\{H^{n}\right\}$ given on some category $K$ of pairs of topological spaces the sequence $\Pi=\left\{\Pi^{n}\right\}, \quad n=0,1,2, \ldots$, of contravariant functors $\Pi^{n}$ is constructed from $K$ into the category of abelian groups with the coboundary operator $\delta^{\#}$ which commutes with the induced homomorphisms $\varphi^{\#}, \varphi \in K$. Functors $\Pi^{n}$ possess the properties of semi-exactness and homotopy and are connected with $H$ by the natural transformations $d: H^{n} \rightarrow \Pi^{n}$ which are the natural equivalences on a certain subcategory $K_{n}$ of $K$. Constructing such functors is reduced to the problem of extending the functor given on an auxiliary subcategory $K_{n}$ to the whole category $K$. The problem is solved by means of the theory of inverse systems of groups with sets of homomorphisms of Hurewiz, Dugundji, and Dowker [4].

Functors $\Pi^{n}$ are dual to the homotopy functors associated in the sense of Bauer [1] with a given homological structure. It should be noted that functors $\Pi^{n}$ have some of the basic properties of the Borsuk cohomotopy, but they differ from the latter.

In [5-7] functors $\Pi^{n}$ were investigated under the assumption that $K$ is the category of pairs of topological spaces with a base point and base point preserving maps, and $H$ is the singular integral theory of cohomology. We will adhere to the same assumption throughout this paper (base points are not indicated here). To define functors $\Pi^{n}$ we need auxiliary subcategories $K_{n}$. We consider the problem of choosing these subcategories.

1991 Mathematics Subject Classification. 55Q55. 
For convenience we recall the definition of a limit of the inverse system of groups with sets of homomorphisms (see [4]). Let $\omega$ be a partially ordered set, and $\left\{G_{\sigma}\right\}$ a system of abelian groups indexed by the elements of $\omega$. Furthermore, let, for each pair $\rho<\sigma$, sets $H_{\sigma \rho} \subset \operatorname{Hom}\left(G_{\sigma}, G_{\rho}\right)$ be given such that if $\rho<\sigma<\tau$ and $\varphi_{1} \in H_{\sigma \rho}, \varphi_{2} \in H_{\tau \sigma}$, then the composition $\varphi_{1} \varphi_{2} \in H_{\tau \rho}$. Then, by definition, $\lim G_{\sigma}$ is a subgroup of the group $\Pi G_{\sigma}$ and its elements are elements $g=\left\{g_{\sigma}\right\} \in \Pi G_{\sigma}$ such that for each pair $\rho<\sigma$ and $\varphi \in H_{\sigma \rho}$ we have $\varphi\left(g_{\sigma}\right)=g_{\rho}$.

It should be noted that this theory of [4] is essentialy Kan's extension theory in its early stage, but quite sufficient for our purpose.

The results of this paper were announced earlier in $[6,7]$.

\section{Preliminaries}

In this section we will give the definitions of subcategories $K_{n}$ and functors $\Pi^{n}$ and discuss some of their properties.

Let $e^{m}$ be the unit $m$-cell of the $m$-dimensional euclidean space $\mathbb{R}^{m}$. By $e^{0}$ we denote some fixed point (base point). Let $\widetilde{K}$ be the small full subcategory of $K$ whose objects are all finite $C W$-complexes $X$ for which $X^{0}=e^{0}$ and $X^{k}$ is the adjunction space obtained by adjoining a finite number of $e^{k}$ to $X^{k-1}, k>0$. We denote by $\widetilde{\widetilde{K}}$ the small full subcategory of $K$ whose objects are all $C W$-pairs $\left(X, X^{\prime}\right)$ for which $X$ and $X^{\prime}$ are the objects of $\widetilde{K}$.

Now we shall define auxiliary subcategories $K_{n}, n>3$, by the following two conditions (cf. $[2,5])$ :

1) $K_{n}$ is an arbitrary small full subcategory of $K$; each object of $K_{n}$ is a pair $\left(X, X^{\prime}\right)$ of linearly and simply connected spaces satisfing the conditions that $\pi_{2}\left(X, X^{\prime}\right)=1$, the homology modules $H_{*}(X)$ and $H_{*}\left(X^{\prime}\right)$ are of finite type, $H^{i}\left(X, X^{\prime}\right)=0, i<n$, and $H^{i}(X)=H^{i}\left(X^{\prime}\right)=0,0<i<n-1$;

2) $K_{n}$ contains all possible objects of $\widetilde{K}$.

We denote by $F_{n}^{r}$ an auxiliary subcategory of objects only of $\widetilde{\widetilde{K}}$.

If $n=3$, we assume that $K_{3}$ is an arbitrary (containing all possible objects of $\widetilde{K}$ ) small full subcategory of $K$ whose all objects are linearly, and simply connected spaces $X$ for which $H_{*}(X)$ is a module of finite type and $H^{2}(X)=0$.

Let $\left(R, R^{\prime}\right)$ be an object of $K$. Consider a set of indices $\omega\left(R, R^{\prime} ; n\right)$ of all pairs $\alpha=\left(X, X^{\prime} ; f\right)$, where $\left(X, X^{\prime}\right)$ is an object of $K_{n}$ and $f:\left(X, X^{\prime}\right) \rightarrow$ $\left(R, R^{\prime}\right)$ is a continuous map of $K$. Let $\omega\left(R, R^{\prime} ; n\right)$ be ordered as follows: $\alpha<\beta$, where $\beta=\left(Y, Y^{\prime} ; g\right)$ if there is a map $\varphi:\left(X, X^{\prime}\right) \rightarrow\left(Y, Y^{\prime}\right)$ of $K_{n}$ such that

$$
g \varphi=f
$$


Assume that to every $\alpha \in \omega\left(R, R^{\prime} ; n\right)$ there corresponds the $n$-dimensional cohomology group $H_{\alpha}=H^{n}\left(X, X^{\prime}\right)$ and to every ordered pair $\alpha<\beta$ there corresponds the set of homomorphisms $\left\{\varphi^{*}\right\}$, where $\varphi^{*}: H^{n}\left(Y, Y^{\prime}\right) \rightarrow$ $H^{n}\left(X, X^{\prime}\right)$ are the induced homomorphisms in the $H$ theory.

We have obtained the inverse system of the group $H_{\alpha}$ with sets of homomorphisms. Cohomotopic groups of Chogoshvili are determined by the formula $\Pi^{n}\left(R, R^{\prime} ; K_{n}\right)=\lim _{\leftarrow} H_{\alpha}$.

We denote by $\Pi^{n}\left(R ; K_{n}\right)$ the absolute group $\Pi^{n}\left(R, * ; K_{n}\right)$, where $*$ is a base point, and by $p_{\alpha}$ the $\alpha$-coordinate of an element $p \in \Pi^{n}\left(R, R^{\prime} ; K_{n}\right)$. Note that for $n=3$ we have determined the absolute groups only.

Let

$$
\begin{gathered}
\alpha=\left(X, X^{\prime} ; f\right), \quad \beta=\left(X, X^{\prime} ; g\right), \\
\alpha, \beta \in \omega\left(R, R^{\prime} ; n\right), \quad p \in \Pi^{n}\left(R, R^{\prime} ; K_{n}\right)
\end{gathered}
$$

and let the maps $f$ and $g$ be homotopic, i.e., $f \sim g$. Let $I$ be the unit segment.

Lemma 1.1. If the subcategory $K_{n}$ contains, alongside with $\left(X, X^{\prime}\right)$, the pair $\left(X \times I, X^{\prime} \times I\right)$, then $p_{\alpha}=p_{\beta}$.

Proof. See [5], p. 83.

Let

$$
\begin{gathered}
\alpha=\left(X, X^{\prime} ; f\right), \quad \beta=\left(Y, Y^{\prime} ; g\right), \\
\alpha, \beta \in \omega\left(R, R^{\prime} ; n\right), \quad p \in \Pi^{n}\left(R, R^{\prime} ; K_{n}\right) .
\end{gathered}
$$

Moreover, let us have a map $\varphi:\left(X, X^{\prime}\right) \rightarrow\left(Y, Y^{\prime}\right)$ from $K_{n}$ such that the maps $g \varphi$ and $f$ are homotopic, i.e.,

$$
g \varphi \sim f .
$$

Lemma 1.2. If the subcategory $K_{n}$ contains, alongside with $\left(X, X^{\prime}\right)$, the pair $\left(X \times I, X^{\prime} \times I\right)$, then $\varphi^{*}\left(p_{\beta}\right)=p_{\alpha}$.

Proof. Consider the index $\left(X, X^{\prime} ; g \varphi\right)=\alpha_{1}<\beta$ and apply Lemma 1.1 to $\alpha_{1}$ and $\alpha$.

Let $\alpha=\left(X, X^{\prime} ; f\right)$, where $f$ is null-homotopic, and $p \in \Pi^{n}\left(R, R^{\prime} ; K_{n}\right)$.

Corollary 1.3. If the subcategory $K_{n}$ contains, alongside with $\left(X, X^{\prime}\right)$, the pair $\left(X \times I, X^{\prime} \times I\right)$, then $p_{\alpha}=0$. 
Proof. Apply Lemma 1.2 to the homotopy commutative diagram

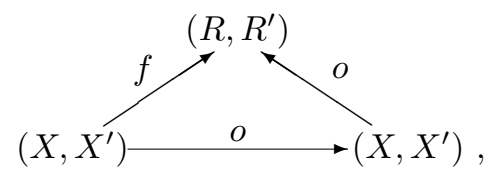

where $o$ denotes the constant map.

\section{MAin Results}

Let $K_{n}^{\prime}$ be a subcategory of $K_{n}^{\prime \prime}$, where $K_{n}^{\prime}$ and $K_{n}^{\prime \prime}$ are two auxiliary subcategories, and let $\left(R, R^{\prime}\right) \in K, \quad p \in \Pi^{n}\left(R, R^{\prime} ; K_{n}^{\prime \prime}\right), \alpha \in{ }^{\prime} \omega\left(R, R^{\prime} ; n\right) \subset$ " $\omega\left(R, R^{\prime} ; n\right)$. Then, as one can easily verify, the formula $[\lambda(p)]_{\alpha}=p_{\alpha}$ defines the restriction homomorphism $\lambda: \Pi^{n}\left(R, R^{\prime} ; K_{n}^{\prime \prime}\right) \rightarrow \Pi^{n}\left(R, R^{\prime} ; K_{n}^{\prime}\right)$.

In Section 3 we will prove

Theorem 2.1. The homomorphism $\lambda$ defines the natural equivalence of the functors $\Pi^{n}\left(-,-; K_{n}^{\prime \prime}\right)$ and $\Pi^{n}\left(-,-; K_{n}^{\prime}\right), n>3$. In particular, all functors $\Pi^{n}\left(-,-; K_{n}\right)$ are naturally equivalent to the functor $\Pi^{n}\left(-,-; F_{n}^{r}\right)$.

Remark 2.2. Theorem 2.1 shows that in choosing a subcategory $K_{n}$ we can restrict ourselves only to the finite $C W$-pairs. On the other hand, from Theorem 2.1 it follows that for the convenience of construction and proof we can regard an arbitrary admissible pair as an object of $K_{n}$.

Remark 2.3. One can easily show that Theorem 2.1 holds for the absolute groups when $n>2$. Moreover, in defining the absolute groups, to choose the subcategory $K_{n}$ we can restrict ourselves to the absolute pairs $(X, *)$, i.e., $X^{\prime}=*$ (see the definition of $K_{n}$ and [5]).

In the remainder of this section we will consider the absolute groups only. Therefore to define the functors $\Pi^{n}, n>2$, we can use auxiliary subcategories $F_{n}^{a}$ consisting of finite $C W$-complexes. More exactly, $F_{n}^{a}$ is a full subcategory $\widetilde{K}$ whose objects are all spaces $X$ for which $\pi_{1}(X)=1$ and $H^{2}(X)=\cdots=H^{n-1}(X)=0$.

We intend here to study the problem dealing with the possibility of further reducing subcategories $K_{n}$ provided that groups $\Pi^{n}\left(R ; K_{n}\right)$ and the results from [5-7] remain unchanged. To this effect, relying on Lemma 1.2, in the definition of $\Pi^{n}\left(R ; K_{n}\right)$ we replace condition (1) by condition (2) (see Section 1). We will stick to this definition in the sequel.

Let $S^{n}$ denote the $n$-dimensional unit sphere of the euclidean space $\mathbb{R}$ and $e^{n}$ the unit disk. Denote by $P^{n}(t), t>1, n>2$, the Moore space $S^{n-1} \cup_{t} e^{n}$. Also assume that $P^{n}(1)=S^{n}$.

Consider now the full subcategory $F_{n}^{b}$ of $F_{n}^{a}$ whose objects are all finite one-point unions of spaces $P^{n}(t), t \geq 1$. The subcategory $F_{n}^{b}$ will be regarded as an auxiliary subcategory. 
The following theorem will be proved in Section 4 .

Theorem 2.4. The restriction homomorphism defines the natural equivalence of the functors $\Pi^{n}\left(-; F_{n}^{a}\right)$ and $\Pi^{n}\left(-; F_{n}^{b}\right), n>2$.

We introduce the following notations:

1) $P_{j}^{n}(t)=P^{n}(t)$, where $j$ is a positive integer, $n>2, t \geq 1$;

2) $X_{k}^{n}=\vee_{t=1}^{k}\left(\vee_{j=1}^{k} P_{j}^{n}(t)\right)$;

3) $Q^{n}=\lim X_{k}$ (by inclusion maps $X_{k}^{n} \rightarrow X_{k+1}^{n}$ ).

Let $Q_{n}$ be the full subcategory of $K$ consisting of one object $Q^{n}, n>2$. The subcategory will also be regarded as an auxiliary subcategory. Note that the module $H_{*}\left(Q^{n}\right)$ is not obviously of the finite type. Therefore none of the above-defined auxiliary subcategories contains $Q^{n}$.

The following theorem will be proved in Section 5 .

Theorem 2.5. The functors $\Pi^{n}\left(-; F_{n}^{b}\right)$ and $\Pi^{n}\left(-; Q_{n}\right)$ are naturally equivalent, $n>2$.

\section{Proof of Theorem 2.1}

Let us prove that $\lambda$ is natural. Assume $f:\left(S, S^{\prime}\right) \rightarrow\left(R, R^{\prime}\right)$ to be an arbitrary map of $K$. Consider the diagram

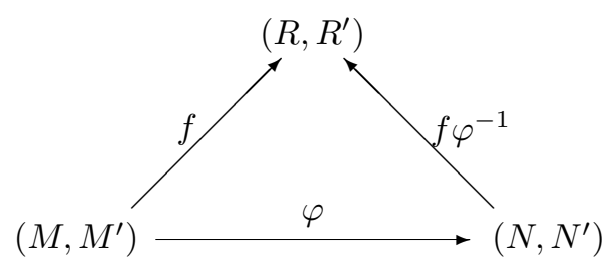

Let

$$
\begin{gathered}
\alpha=\left(X, X^{\prime} ; g\right) \in{ }^{\prime} \omega\left(S, S^{\prime}, n\right) \subset{ }^{\prime \prime} \omega\left(S, S^{\prime} ; n\right), \\
\beta=f(\alpha)=\left(X, X^{\prime} ; f g\right) \in{ }^{\prime} \omega\left(R, R^{\prime} ; n\right) \subset^{\prime \prime} \omega\left(R, R^{\prime} ; n\right)
\end{gathered}
$$

and let $p \in \Pi^{n}\left(R, R^{\prime} ; K_{n}^{\prime \prime}\right)$. We have

$$
\begin{aligned}
& {\left[\lambda\left(f^{\#}(p)\right)\right]_{\alpha}=\left[f^{\#}(p)\right]_{\alpha}=p_{\beta},} \\
& {\left[f^{\#}(\lambda(p))\right]_{\alpha}=[\lambda(p)]_{\beta}=p_{\beta},}
\end{aligned}
$$

which proves that $\lambda$ is natural.

Let $\left(X, X^{\prime}\right)$ be an arbitrary object of some auxiliary subcategory $K_{n}$. Using the standard technique of the homotopy theory, we can construct a $C W$-pair $(A, B)$ from the subcategory $K_{n}$ and a map $\varphi:(A, B) \rightarrow\left(X, X^{\prime}\right)$ such that homomorphisms $\varphi^{*}$ induced by $\varphi$ in the $H$ theory will be isomorphisms up to any pregiven dimension. Let now $p \in \Pi^{n}\left(R, R^{\prime} ; K_{n}\right)$ and $\alpha=$ 
$\left(X, X^{\prime} ; f\right) \in \omega\left(R, R^{\prime} ; n\right)$. Consider the index $\beta=(A, B ; f \varphi) \in \omega\left(R, R^{\prime} ; n\right)$. Then $\beta<\alpha$ and therefore $p_{\beta}=\varphi^{*}\left(p_{\alpha}\right)$. Hence $p_{\alpha}=\varphi^{*-1}\left(p_{\beta}\right)$. From the above reasoning and the definition of auxiliary subcategories it now follows that if $p \in \Pi^{n}\left(R, R^{\prime} ; K_{n}^{\prime \prime}\right)$ and $\lambda(p)=0$, then $p=0$. Thus $\lambda$ is a monomorphism.

Let $L_{n}, n>3$, be a full subcategory of the category $K$ whose objects are all pairs $\left(X, X^{\prime}\right)$ for which $X$ and $X^{\prime}$ are linearly and simply connected spaces, $\pi_{2}\left(X, X^{\prime}\right)=1$, the homology modules $H_{*}(X)$ and $H_{*}\left(X^{\prime}\right)$ are of the finite type, $H^{i}\left(X, X^{\prime}\right)=0, i<n$, and $H^{i}(X)=H^{i}\left(X^{\prime}\right)=0,0<i<n-1$.

Consider some full subcategories of $L_{n}$ :

1) $L_{n}^{(1)}$ are $C W$-pairs;

2) $L_{n}^{(2)}$ are $C W$-pairs with a finite number of cells in all demensions;

3) $L_{n}^{(3)}$ are finite $C W$-pairs;

4) $L_{n}^{(4)}=\widetilde{\widetilde{K}} \cap \mathrm{L}_{n}^{(3)}=F_{n}^{r}$.

We will gradually extend the thread defining element $p \in \Pi^{n}\left(R, R^{\prime} ; F_{n}^{r}\right)$ from the category $L_{n}^{(4)}$ onto $L_{n}^{(3)}$, then onto $L_{n}^{(2)}, L_{n}^{(1)}$ and, finally, onto $L_{n}$. Such an extension already implies that $\lambda$ is epimorphic.

Let $\left(M, M^{\prime}\right)$ be an arbitrary object of $L_{n}^{(3)}$; also let $f:\left(M, M^{\prime}\right) \rightarrow\left(R, R^{\prime}\right)$ be an arbitrary map. Consider the diagram

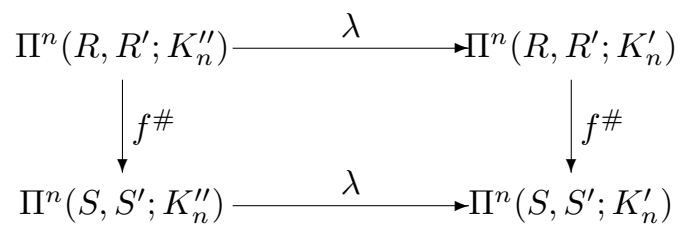

where $\left(N, N^{\prime}\right) \in L_{n}^{(4)}$ and $\varphi$ is a homeomorphism. Let $\alpha=\left(M, M^{\prime} ; f\right)$ and $\beta=\left(N, N^{\prime} ; f \varphi^{-1}\right)$. It is assumed that $p_{\alpha}=\varphi^{*}\left(p_{\beta}\right)$. We will show that $p_{\alpha}$ does not depend on the choice of the homeomorphism $\varphi$. Consider the diagram

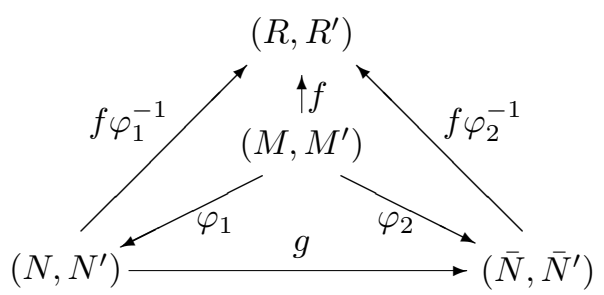

where $\varphi_{1}$ and $\varphi_{2}$ are two different homeomorphisms and $g=\varphi_{2} \varphi_{1}^{-1}$. The indices $\beta_{1}$ and $\beta_{2}$ will be defined similarly to $\beta$. We have $\left(f \varphi_{2}^{-1}\right) g=$ $f \varphi_{2}^{-1} \varphi_{2} \varphi_{1}^{-1}=f \varphi_{1}^{-1}$. Therefore $\beta_{1}<\beta_{2}$. Then $\varphi_{2}^{*}\left(p_{\beta_{2}}\right)=\left(g \varphi_{1}\right)^{*}\left(p_{\beta_{2}}\right)=$ $\varphi_{1}^{*}\left(g^{*}\left(p_{\beta_{2}}\right)\right)=\varphi_{1}^{*}\left(p_{\beta_{1}}\right)$. 
Consider the commutative diagram

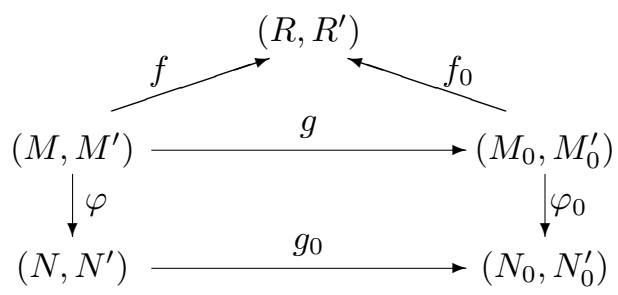

where $\varphi$ and $\varphi_{0}$ are homeomorphisms, $g_{0}=\varphi_{0} g \varphi^{-1}$.

Let

$$
\begin{aligned}
\alpha=\left(M, M^{\prime} ; f\right), & \alpha_{0}=\left(M_{0}, M_{0}^{\prime} ; f_{0}\right), \\
\beta=\left(N, N^{\prime} ; f \varphi^{-1}\right), & \beta_{0}=\left(N_{0}, N_{0}^{\prime} ; f_{0} \varphi_{0}^{-1}\right) .
\end{aligned}
$$

We have

$$
\left(f_{0} \varphi_{0}^{-1}\right) g_{0}=f_{0} \varphi_{0}^{-1} \varphi_{0} g \varphi^{-1}=f_{0} g \varphi^{-1}=f \varphi^{-1} .
$$

Therefore $\beta<\beta_{0}$. Then

$$
\begin{gathered}
g^{*}\left(p_{\alpha_{0}}\right)=g^{*}\left(\varphi_{0}^{*}\left(p_{\beta_{0}}\right)\right)=\left(\varphi_{0} g\right)^{*}\left(p_{\beta_{0}}\right)=\left(g_{0} \varphi\right)^{*}\left(p_{\beta_{0}}\right)= \\
=\varphi^{*}\left(g_{0}^{*}\left(p_{\beta_{0}}\right)\right)=\varphi^{*}\left(p_{\beta}\right)=p_{\alpha} .
\end{gathered}
$$

We have thus extended the thread of the element $p$ onto the category $L_{n}^{(3)}$.

Let, now, $\left(M, M^{\prime}\right) \in L_{n}^{(2)}$ and $f:\left(M, M^{\prime}\right) \rightarrow\left(R, R^{\prime}\right)$ be an arbitrary map. By $i: X_{k} \rightarrow X$ we denote here the standard embedding, where $X_{k}$ is the $k$-skeleton of the $C W$-complex $X$. Let $N_{1}>N>n+1$ be arbitrary integers.

Consider the commutative diagram

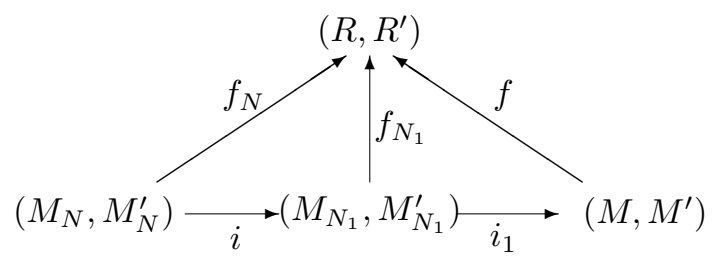

where $f_{N_{1}}=f i_{1}$ and $f_{N}=f_{N_{1}} i$. Also consider the indices

$$
\alpha=\left(M_{N}, M_{N}^{\prime} ; f_{N}\right), \quad \beta=\left(M_{N_{1}}, M_{N_{1}}^{\prime} ; f_{N_{1}}\right), \quad \gamma=\left(M, M^{\prime} ; f\right) .
$$

Assume $p_{\gamma}=i_{1}^{*-1}\left(p_{\beta}\right)$. We have

$$
\left(i_{1} i\right)^{*-1}\left(p_{\alpha}\right)=i_{1}^{*-1}\left(i^{*-1}\left(p_{\alpha}\right)\right)=i_{1}^{*-1}\left(p_{\beta}\right),
$$

where the last equality evidently follows from the fact that $\alpha<\beta$. Therefore $p_{\gamma}$ does not depend on the choice of the number $N_{1}$. 
Now consider the diagram

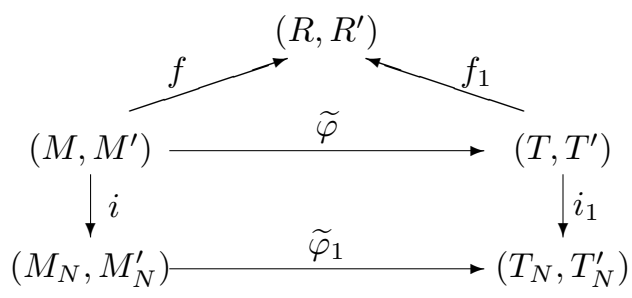

where $\varphi:\left(M, M^{\prime}\right) \rightarrow\left(T, T^{\prime}\right)$ is a map such that $f_{1} \varphi=f, \widetilde{\varphi}$ is a cellular approximation of $\varphi$ and $\widetilde{\varphi_{1}}=\widetilde{\varphi} \mid\left(M_{N}, M_{N}^{\prime}\right)$. We have $f \sim f_{1} \widetilde{\varphi}$ and $\left(f_{1} i_{1}\right) \widetilde{\varphi_{1}}=\left(f_{1} \widetilde{\varphi}\right) i \sim f i$. Also consider the indices $\alpha=\left(M_{N}, M_{N}^{\prime} ; f i\right), \beta=$ $\left(T_{N}, T_{N}^{\prime} ; f_{1} i_{1}\right)$. Applying Lemma 1.2 , we have $\varphi^{*}\left(i_{1}^{*-1}\left(p_{\beta}\right)\right)=\left(\bar{\varphi}^{*} i_{1}^{*-1}\right)\left(p_{\beta}\right)$ $=i^{*-1}\left(\widetilde{\varphi_{1}}{ }^{*}\left(p_{\beta}\right)\right)=i^{*-1}\left(p_{\alpha}\right)$.

We have thus extended the thread of the element $p$ onto the category $L_{n}^{(2)}$.

Let now $\left(\bar{M}, \bar{M}^{\prime}\right)$ be an arbitrary object of $L_{n}^{(1)}$ and let $\bar{g}:\left(\bar{M}, \bar{M}^{\prime}\right) \rightarrow$ $\left(R, R^{\prime}\right)$ be an arbitrary map. Using the standard technique of the homotopy theory, we can, under our assumptions, construct a map $\varphi:\left(M, M^{\prime}\right) \rightarrow$ $\left(\bar{M}, \bar{M}^{\prime}\right)$ such that $\left(M, M^{\prime}\right) \in L_{n}^{(2)}$ and $\varphi$ is a homotopy equivalence. Let $g=\bar{g} \varphi$. Assume $p_{\alpha}=\varphi^{*-1}\left(p_{\beta}\right)$, where $\alpha=\left(\bar{M}, \bar{M}^{\prime} ; \bar{g}\right), \beta=\left(M, M^{\prime} ; g\right)$. We will show that $p_{\alpha}$ does not depend on the choice of $\varphi$. Consider the diagram

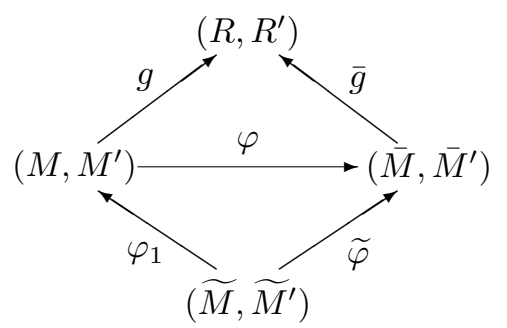

where $\left(\widetilde{M}, \widetilde{M}^{\prime}\right) \in L_{n}^{(2)}, \widetilde{\varphi}$ and $\varphi_{1}$ are homotopy equivalences, and $\varphi \sim \widetilde{\varphi} \varphi_{1}$. Let $\widetilde{\beta}=\left(\widetilde{M}, \widetilde{M}^{\prime} ; \bar{g} \widetilde{\varphi}\right)$. Then

$$
g=\bar{g} \varphi \sim \bar{g} \widetilde{\varphi} \varphi_{1}=(\bar{g} \widetilde{\varphi}) \varphi_{1} .
$$

Applying Lemma 1.2, we have

$$
\widetilde{\varphi}^{*-1}\left(p_{\widetilde{\beta}}\right)=\widetilde{\varphi}^{*-1}\left(\varphi_{1}^{*-1}\left(p_{\beta}\right)\right)=\varphi^{*-1}\left(p_{\beta}\right) .
$$

Consider now an arbitrary map

$$
\varphi_{0}:\left(\bar{M}, \bar{M}^{\prime}\right) \rightarrow\left(\bar{T}, \bar{T}^{\prime}\right)
$$


from the category $L_{n}^{(1)}$ and the diagram

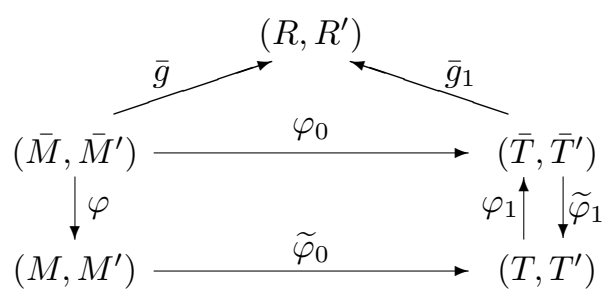

where $\bar{g}=\overline{g_{1}} \varphi_{0}, \varphi$ and $\varphi_{1}$ are homotopy equivalences, $\widetilde{\varphi}_{1}$ is the homotopy inverse of $\varphi_{1}, \widetilde{\varphi_{0}}=\widetilde{\varphi_{1}} \varphi_{0} \varphi$ and $\left(M, M^{\prime}\right),\left(T, T^{\prime}\right) \in L_{n}^{(2)}$. We have $\left(\overline{g_{1}} \varphi_{1}\right) \widetilde{\varphi_{0}}=\overline{g_{1}} \varphi_{1} \widetilde{\varphi_{1}} \varphi_{0} \varphi \sim \overline{g_{1}} \varphi_{0} \varphi=\bar{g} \varphi$. Also, consider the indices $\beta=$ $\left(M, M^{\prime} ; \bar{g} \varphi\right), \quad \beta_{1}=\left(T, T^{\prime} ; \overline{g_{1}} \varphi_{1}\right)$. Then, applying Lemma 1.2 , we have $\varphi_{0}^{*}\left(\varphi_{1}^{*-1}\left(p_{\beta_{1}}\right)\right)=\left(\varphi_{0}^{*} \varphi_{1}^{*-1}\right)\left(p_{\beta_{1}}\right)=\left(\varphi^{*-1}{\widetilde{\varphi_{0}}}^{*}\right)\left(p_{\beta_{1}}\right)=\varphi^{*-1}\left(p_{\beta}\right)$.

Let, finally, $\left(X, X^{\prime}\right)$ be an arbitrary object of $L_{n}$, and let

$$
w_{X}:\left(S(X), S\left(X^{\prime}\right)\right) \rightarrow\left(X, X^{\prime}\right)
$$

be the natural projection of the singular complex $S(X)$ onto $X$. Let $f$ : $\left(X, X^{\prime}\right) \rightarrow\left(R, R^{\prime}\right)$ be an arbitrary map. Also, consider the indices $\alpha=$ $\left(X, X^{\prime} ; f\right), \beta=\left(S(X), S\left(X^{\prime}\right) ; f w_{X}\right)$. Assume $p_{\alpha}=w_{X}^{*-1}\left(p_{\beta}\right)$ and consider the commutative diagram

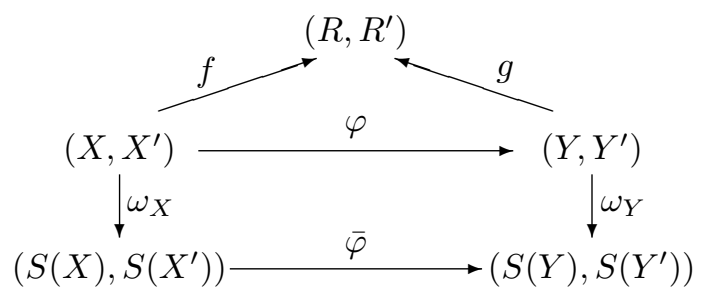

where $g \varphi=f$ and $\bar{\varphi}$ is the cellular map induced by $\varphi$. We have $\left(g w_{Y}\right) \bar{\varphi}=$ $g \varphi w_{X}=f w_{X}$. Also, consider the indices $\beta_{X}=\left(S(X), S\left(X^{\prime}\right) ; f \omega_{X}\right), \beta_{Y}=$ $\left(S(Y), S\left(Y^{\prime}\right) ; g \omega_{Y}\right)$. Then

$$
\varphi^{*}\left(\omega_{Y}^{*-1}\left(p_{\beta_{Y}}\right)\right)=\omega_{X}^{*-1}\left(\bar{\varphi}^{*}\left(p_{\beta_{Y}}\right)\right)=\omega_{X}^{*-1}\left(p_{\beta_{X}}\right) .
$$

This completes the proof of Theorem 2.1.

\section{Proof of Theorem 2.4}

Consider some full subcategories of $F_{n}^{a}$ (see Section 2):

0) $K_{n}^{(0)}=F_{n}^{a}$;

1) $K_{n}^{(1)}$-objects are $C W$-complexes with one vertex and without cells of dimensions $1,2, \ldots, n-2$; 
2) $K_{n}^{(2)}$-objects are $C W$-complexes with one vertex and cells of dimensions $n-1, n$ and $n+1$ only;

3) $K_{n}^{(3)}$-objects are $C W$-complexes with one vertex and cells of dimensions $n-1$ and $n$ only;

4) $K_{n}^{(4)}=F_{n}^{b}$.

Let $R=(R, *)$ be an arbitrary space from $K$. All subcategories $K_{n}^{(i)}$, $0 \leq i \leq 4$, will be regarded as auxiliary ones.

Let

$$
\lambda_{i}: \Pi^{n}\left(R ; K_{n}^{(i)}\right) \rightarrow \Pi^{n}\left(R ; K_{n}^{(i+1)}\right), \quad 0 \leq i \leq 3,
$$

be the natural restriction homomorphisms.

Let $L_{n}^{\prime}$ and $L_{n}^{\prime \prime}$ be two small full subcategories of the category $K$ consisting of the spaces $(X, *)$, and $L_{n}^{\prime} \subset L_{n}^{\prime \prime}$. It is assumed that the following condition is satisfied: for each $X \in L_{n}^{\prime \prime}$ there is $Y \in L_{n}^{\prime}$ such that $Y$ has the same homotopy type as $X$. Consider $L_{n}^{\prime}$ and $L_{n}^{\prime \prime}$ as auxiliary subcategories. Let $\bar{\lambda}: \Pi^{n}\left(R ; L_{n}^{\prime \prime}\right) \rightarrow \Pi^{n}\left(R ; L_{n}^{\prime}\right)$ be the natural restriction homomorphism.

Lemma 4.1. $\bar{\lambda}$ is a natural isomorphism.

Proof. We prove first that the homomorphism $\bar{\lambda}$ is a monomorphism. Let $p \in \Pi^{n}\left(R ; L_{n}^{\prime \prime}\right), \bar{\lambda}(p)=0$ and $\alpha=(X ; f) \in{ }^{\prime \prime} \omega(R ; n)$ be an arbitrary index. Let $Y \in L_{n}^{\prime}$ and the map $\varphi: Y \rightarrow X$ be a homotopy equivalence. Consider the index $\beta=(Y ; f \varphi) \in{ }^{\prime} \omega(R ; n) \subset{ }^{\prime \prime} \omega(R ; n)$. We have $\beta<\alpha$. Then $\varphi^{*}\left(p_{\alpha}\right)=p_{\beta}=[\bar{\lambda}(p)]_{\beta}=0$. Therefore $p_{\alpha}=0$ and $p=0$.

Let us now prove that the homomorphism $\bar{\lambda}$ is an epimorphism. Let $q \in \Pi^{n}\left(R ; L_{n}^{\prime}\right)$ and $\alpha=(X ; f) \in{ }^{\prime \prime} \omega(R ; n)$ be an arbitrary index. Let $Y \in L_{n}^{\prime}$ and the map $\varphi: Y \rightarrow X$ be a homotopy equivalence. Consider the index $\beta=(Y ; f \varphi) \in^{\prime} \omega(R ; n)$ and assume $p_{\alpha}=\varphi^{*-1}\left(q_{\beta}\right)$. We will show that $p_{\alpha}$ does not depend on the choice of $\varphi$. Consider the diagram

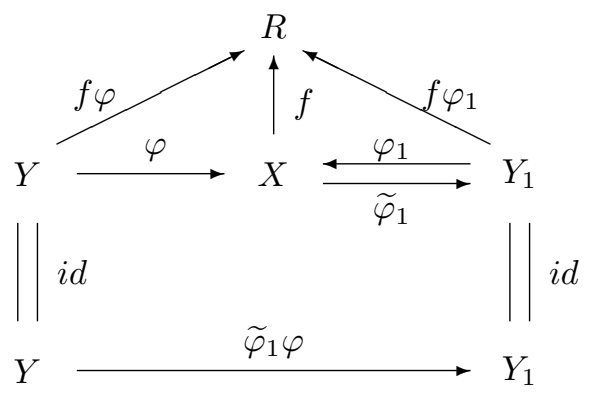

where $Y, Y_{1} \in L_{n}^{\prime}, \varphi$ and $\varphi_{1}$ are homotopy equivalences, and $\widetilde{\varphi}_{1}$ is the homotopy inverse of $\varphi_{1}$. Consider the index $\gamma=\left(Y_{1} ; f \varphi_{1}\right) \in{ }^{\prime} \omega(R ; n)$. 
Then $\left(f \varphi_{1}\right)\left(\widetilde{\varphi}_{1} \varphi\right) \sim f \varphi$ and therefore $\beta<\gamma$. In this case

$$
\begin{aligned}
& \varphi_{1}^{*}\left(\varphi^{*-1}\left(q_{\beta}\right)\right)=\varphi_{1}^{*}\left(\varphi^{*-1}\left(\left(\widetilde{\varphi}_{1} \varphi\right)^{*}\left(q_{\gamma}\right)\right)\right)= \\
& =\left(\varphi_{1}^{*} \varphi^{*-1} \varphi^{*} \widetilde{\varphi}_{1}^{*}\right)\left(q_{\gamma}\right)=\left(\varphi_{1}^{*} \widetilde{\varphi}_{1}^{*}\right)\left(q_{\gamma}\right)=q_{\gamma} .
\end{aligned}
$$

Hence $\varphi^{*-1}\left(q_{\beta}\right)=\varphi_{1}^{*-1}\left(q_{\gamma}\right)$.

We will show that the set $\left\{p_{\alpha}\right\}$ defines an element of the group $\Pi^{n}\left(R ; L_{n}^{\prime \prime}\right)$. Consider the diagram

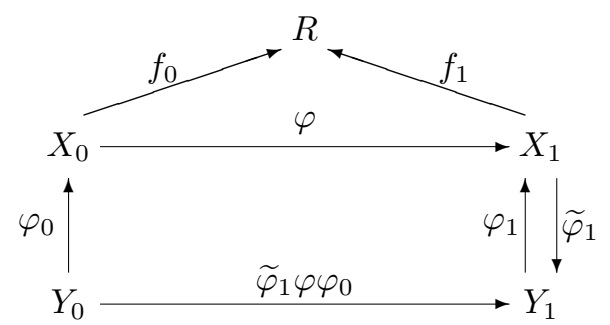

where $f_{1} \varphi=f_{0}, \varphi_{0}$ and $\varphi_{1}$ are homotopy equivalences, $\widetilde{\varphi}_{1}$ is the homotopy inverse of $\varphi_{1}, X_{0}, X_{1} \in L_{n}^{\prime \prime}$, and $Y_{0}, Y_{1} \in L_{n}^{\prime}$. Then

$$
\left(f_{1} \varphi_{1}\right)\left(\widetilde{\varphi}_{1} \varphi \varphi_{0}\right) \sim f_{1} \varphi \varphi_{0}=f_{0} \varphi_{0}
$$

Also, consider the indices

$$
\begin{gathered}
\alpha=\left(X_{0} ; f_{0}\right), \quad \beta=\left(X_{1} ; f_{1}\right), \quad \alpha, \beta \in \in^{\prime \prime} \omega(R ; n), \\
\alpha_{1}=\left(Y_{0} ; f_{0} \varphi_{0}\right), \quad \beta_{1}=\left(Y_{1} ; f_{1} \varphi_{1}\right), \quad \alpha_{1}, \beta_{1} \in^{\prime} \omega(R ; n) \subset{ }^{\prime \prime} \omega(R ; n) .
\end{gathered}
$$

Then $\alpha<\beta, \alpha_{1}<\beta_{1}$ and we have

$$
\varphi^{*}\left(p_{\beta}\right)=\varphi^{*}\left(\varphi_{1}^{*-1}\left(q_{\beta_{1}}\right)\right)=\varphi_{0}^{*-1}\left(\left(\widetilde{\varphi}_{1} \varphi \varphi_{0}\right)^{*}\left(q_{\beta_{1}}\right)\right)=\varphi_{0}^{*-1}\left(q_{\alpha_{1}}\right)=p_{\alpha} .
$$

Finally, let us prove that $\bar{\lambda}(p)=q$. Assume that $X \in L_{n}^{\prime} \subset L_{n}^{\prime \prime}$ and

$$
\alpha=(X ; f) \in^{\prime} \omega(R ; n) \subset{ }^{\prime \prime} \omega(R ; n) .
$$

Define $p_{\alpha}$ by taking $\varphi=i d: X \rightarrow X$. Then

$$
[\bar{\lambda}(p)]_{\alpha}=p_{\alpha}=i d^{*-1}\left(q_{\alpha}\right)=q_{\alpha} .
$$

This completes the proof of Lemma 4.1.

As a consequence of the foregoing lemma we have

Proposition 4.2. $\lambda_{0}$ is a natural isomorphism.

Proof. Every $C W$-complex from $K_{n}^{(0)}$ is homotopically equivalent to some $C W$-complex from $K_{n}^{(1)}$.

Proposition 4.3. $\lambda_{1}$ is a natural isomorphism. 
Proof. We will prove in the first place that $\lambda_{1}$ is a monomorphism. Let $p \in \Pi^{n}\left(R ; K_{n}^{(1)}\right)$ and $\lambda_{1}(p)=0$. Consider the index $\alpha=(X ; f) \in{ }^{(1)} \omega(R ; n)$, $X \in K_{n}^{(1)}$, and the diagram

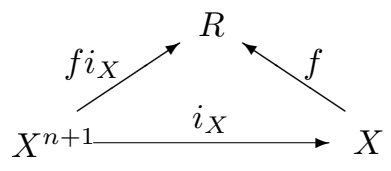

where $X^{n+1}$ is the $(n+1)$-skeleton of $X$ and $i_{X}$ is the standard embedding. Let

$$
\beta=\left(X^{n+1} ; f i_{X}\right) \in{ }^{(2)} \omega(R ; n) \subset{ }^{(1)} \omega(R ; n) .
$$

Then $\beta<\alpha$, and we have $i_{X}^{*}\left(p_{\alpha}\right)=p_{\beta}=\left[\lambda_{1}(p)\right]_{\beta}=0$. Therefore $p=0$.

Assume now that $p \in \Pi^{n}\left(R ; K_{n}^{(2)}\right)$ and $\alpha=(X ; f) \in{ }^{(1)} \omega(R ; n)$.

Consider the diagram (3), the index $\beta$, and assume that $q_{\alpha}=i_{X}^{*-1}\left(p_{\beta}\right)$.

We will show that the set $\left\{q_{\alpha}\right\}$ defines an element of $\Pi^{n}\left(R ; K_{n}^{(1)}\right)$. Consider the diagram

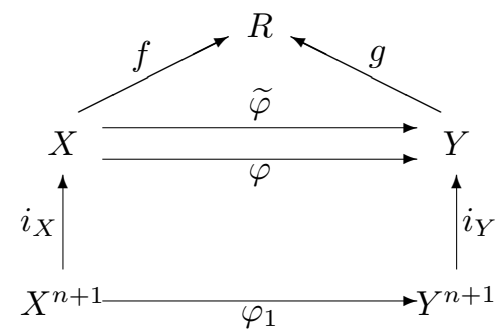

where $g \varphi \sim f, \widetilde{\varphi}$ is a cellular approximation of $\varphi$ and $\varphi_{1}=\widetilde{\varphi} \mid X^{n+1}$. Then $\left(g i_{Y}\right) \varphi_{1}=g \widetilde{\varphi} i_{X} \sim f i_{X}$. Also, consider the indices

$$
\begin{gathered}
\alpha_{1}=(Y ; g) \in{ }^{(1)} \omega(R ; n), \\
\beta_{1}=\left(Y^{n+1} ; g i_{Y}\right) \in{ }^{(2)} \omega(R ; n) .
\end{gathered}
$$

We have $\beta<\beta_{1}$ and

$$
\varphi^{*}\left(q_{\alpha_{1}}\right)=\widetilde{\varphi}^{*}\left(q_{\alpha_{1}}\right)=\widetilde{\varphi}^{*}\left(i_{Y}^{*-1}\left(q_{\beta_{1}}\right)\right)=i_{X}^{*-1}\left(\varphi_{1}^{*}\left(q_{\beta_{1}}\right)\right)=i_{X}^{*-1}\left(q_{\beta}\right)=q_{\alpha} .
$$

Finally, let us prove that $\lambda_{1}(q)=p$.

Consider the index $\alpha=(X ; f) \in{ }^{(2)} \omega(R ; n)$, where $X=X^{n+1} \in K_{n}^{(2)} \subset$ $K_{n}^{(1)}$, and the diagram 


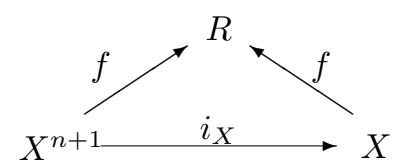

where $i_{X}=i d$. Then $\left[\lambda_{1}(q)\right]_{\alpha}=i_{X}^{*-1}\left(p_{\alpha}\right)=p_{\alpha}$.

This completes the proof of Proposition 4.3.

Proposition 4.4. $\lambda_{2}$ is a natural isomorphism.

Proof. Let $q \in \Pi^{n}\left(R ; K_{n}^{(2)}\right)$ and $\lambda_{2}(q)=0$. Consider the index $\alpha=$ $\left(P_{n+1} ; f\right) \in{ }^{(2)} \omega(R ; n)$, where $P_{n+1} \in K_{n}^{(2)}$. Let $P_{n+1}^{(n)}$ be the $n$-skeleton of $P_{n+1}$ and $i: P_{n+1}^{(n)} \rightarrow P_{n+1}$ be the standard embedding. We have the commutative diagram

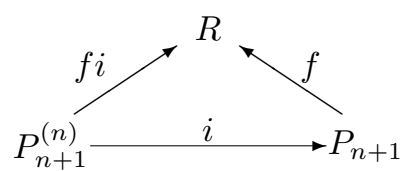

Also consider the index

$$
\beta=\left(P_{n+1}^{(n)} ; f i\right) \in{ }^{(3)} \omega(R ; n) \subset{ }^{(2)} \omega(R ; n) .
$$

We have $\beta<\alpha$. Let

$$
\rightarrow H^{n}\left(P_{n+1}, P_{n+1}^{(n)}\right) \rightarrow H^{n}\left(P_{n+1}\right) \stackrel{i^{*}}{\rightarrow} H^{n}\left(P_{n+1}^{(n)}\right) \rightarrow
$$

be a part of the cohomological exact sequence for the pair $\left(P_{n+1}, P_{n+1}^{(n)}\right)$. Since $H^{n}\left(P_{n+1}, P_{n+1}^{(n)}\right)=0, i^{*}$ is a monomorphism. Then $i^{*}\left(q_{\alpha}\right)=q_{\beta}=$ $\left[\lambda_{2}(q)\right]_{\beta}=0$. Therefore $q_{\alpha}=0$ and $q=0$. Hence $\lambda_{2}$ is a monomorphism.

Let now $q \in \Pi^{n}\left(R ; K_{n}^{(3)}\right)$. Consider again the commutative diagram (4) and the corresponding indices $\alpha$ and $\beta$. We will prove below that $q_{\beta} \in \operatorname{Im} i^{*}$. Therefore $p_{\alpha}=i^{*-1}\left(q_{\beta}\right)$ is the correct definition. Let us show that the set $\left\{p_{\alpha}\right\}$ defines an element $p \in \Pi^{n}\left(R ; K_{n}^{(2)}\right)$. Consider the diagram

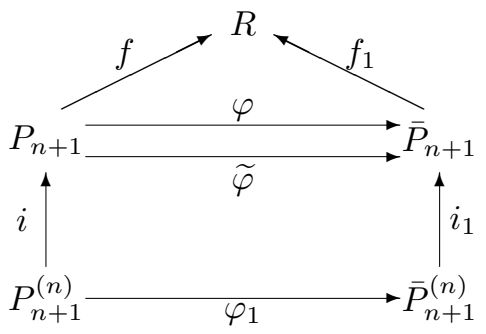

where $f_{1} \varphi \sim f, \widetilde{\varphi}$ is a cellular approximation of $\varphi$ and $\varphi_{1}=\widetilde{\varphi} \mid p_{n+1}^{(n)}$. Con- 
sider the indices

$$
\alpha_{1}=\left(\bar{p}_{n+1} ; f_{1}\right) \in{ }^{(2)} \omega(R ; n), \quad \beta_{1}=\left(\bar{p}_{n+1}^{(n)} ; f_{1} i_{1}\right) \in{ }^{(3)} \omega(R ; n) .
$$

Since $\left(f_{1} i_{1}\right) \varphi_{1}=f_{1} \widetilde{\varphi} i \sim f i$, we obtain $\beta<\beta_{1}$ and therefore $\varphi_{1}^{*}\left(q_{\beta_{1}}\right)=q_{\beta}$. We have $i^{*-1}\left(\varphi_{1}^{*}\left(q_{\beta_{1}}\right)\right)=\widetilde{\varphi}^{*}\left(i_{1}^{*-1}\left(q_{\beta_{1}}\right)\right)$ and

$$
\varphi^{*}\left(p_{\alpha_{1}}\right)=\widetilde{\varphi}^{*}\left(p_{\alpha_{1}}\right)=\widetilde{\varphi}^{*}\left(i_{1}^{*-1}\left(q_{\beta_{1}}\right)\right)=i^{*-1}\left(\varphi_{1}^{*}\left(q_{\beta_{1}}\right)\right)=i^{*-1}\left(q_{\beta}\right)=p_{\alpha} .
$$

Finally, we will prove that $\lambda_{2}(p)=q$. Let

$$
\alpha=\left(P_{n+1} ; f\right) \in{ }^{(3)} \omega(R ; n) \subset{ }^{(2)} \omega(R ; n)
$$

be an arbitrary index, $P_{n+1} \in K_{n}^{(3)}$. Thus $P_{n+1}^{(n)}=P_{n+1}$. Then the map $i: P_{n+1}^{(n)} \rightarrow P_{n+1}$ is the identity map: $i=i d$. Therefore for $\beta=\left(P_{n+1}^{(n)} ; f i\right)$ we have $\beta=\alpha$. In this case

$$
\left[\lambda_{2}(p)\right]_{\alpha}=p_{\alpha}=i^{*-1}\left(q_{\beta}\right)=i d^{*-1}\left(q_{\alpha}\right)=q_{\alpha} .
$$

It remains to prove that $q_{\beta} \in \operatorname{Im} i^{*}$. Consider the characteristic map of the $C W$-complex $P_{n+1}$

$$
\Phi:\left(C\left(\vee S^{n}\right), \vee S^{n}\right) \rightarrow\left(P_{n+1}, P_{n+1}^{(n)}\right),
$$

where $C\left(\vee S^{n}\right)$ denotes the cone over $\vee S^{n}$ and $\vee$ denotes the finite one-point union of spaces. Let $\varphi=\Phi \mid\left(\vee S^{n}\right)$. Consider the commutative diagram

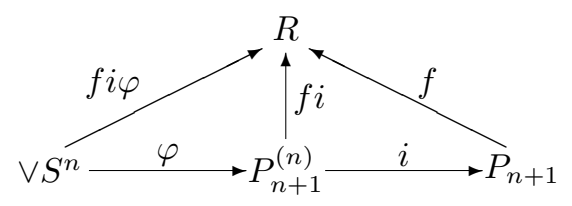

Since $i \varphi \sim 0$, we obtain $f i \varphi \sim 0$. Let $\gamma=\left(\vee S^{n} ; f i \varphi\right) \in{ }^{(3)} \omega(R ; n)$. We have $\gamma<\beta$ and $q_{\gamma}=0$ (see the proof of Corollary 1.3). In this case $\varphi^{*}\left(q_{\beta}\right)=q_{\gamma}=0$.

Now consider the commutative diagram

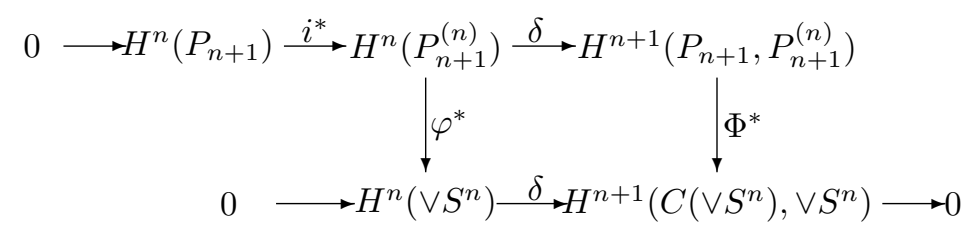

where $\Phi^{*}$ is an isomorphism. Then, since $\varphi^{*}\left(q_{\beta}\right)=0$, we have $\delta\left(q_{\beta}\right)=0$. Therefore $q_{\beta} \in \operatorname{Im} i^{*}$. This completes the proof of Proposition 4.4. 
Lemma 4.5. For each $C W$-complex $P$ from the category $K_{n}^{(3)}$ there is a $C W$-complex $\bar{P}$ from the category $K_{n}^{(4)}$ such that $\bar{P}$ has the homotopy type of $P$.

Proof. By the condition $H_{i}(P)=0, i \neq 0, n-1, n ; H_{n-1}(P) \approx \pi_{n-1}(P)$ are finite abelian groups and $H_{n}(P)$ is a finitely generated free abelian group. Thus the group $\pi_{n-1}(P)$ can be represented in the form

$$
\pi_{n-1}(P) \approx Z_{r_{1}} \oplus Z_{r_{2}} \oplus \cdots \oplus Z_{r_{t}},
$$

where $Z_{r_{i}}, i=1,2, \ldots, t$, are cyclic groups of order $r_{i}$. Consider the corresponding system $\xi_{i}: S^{n-1} \rightarrow P, 1 \leq i \leq t$, of generators in the group $\pi_{n-1}(P)$ and define, by means of $\xi_{i}$, the map $f: \vee_{i=1}^{t} P^{n}\left(r_{i}\right) \rightarrow P$. Then $f$ induces isomorphisms in homotopy and homology in dimensions $\leq n-1$. Now consider a system $h_{1}, h_{2}, \ldots, h_{s}$ of generators in the group $H_{n}(P)$. The Hurewicz homomorphism $\pi_{n}(P) \rightarrow H_{n}(P)$ for the space $P$ is an epimorphism. In this case we can consider maps $\varphi_{k}: S^{n} \rightarrow P, k=1,2, \ldots, s$, such that $\varphi_{k *}(1)=h_{k}$, where $1 \in H_{n}\left(S^{n}\right)$. Assume

$$
\bar{P}=\left(\bigvee_{i=1}^{t} P^{n}\left(r_{i}\right)\right) \bigvee\left(\bigvee_{k=1}^{s} P_{k}^{n}(1)\right),
$$

where $P_{k}^{n}(1)=P^{n}(1)=S^{n}$, and define by means of the maps $f$ and $\varphi_{k}$ the map $\varphi=f \vee\left(\vee \varphi_{k}\right): \bar{P} \rightarrow P$. Then $\varphi$ induces isomorphisms of all homology groups. Therefore, under our assumptions, the map $\varphi$ is a homotopy equivalence. This proves Lemma 4.5.

Lemmas 4.1 and 4.5 imply

Proposition 4.6. $\lambda_{3}$ is a natural isomorphism.

Propositions 4.2 - 4.4 and 4.6 imply Theorem 2.4.

$$
\text { 5. Proof of Theorem } 2.5
$$

Let $h \in H^{n}\left(Q^{n}\right)$ and $i_{j, t}: P^{n}(t) \rightarrow Q^{n}$ be standard embeddings. Assume

$$
\varepsilon(h)=\left\{i_{j, t}^{*}(h)\right\} \in \prod_{j, t} H^{n}\left(P_{j}^{n}(t)\right) .
$$

Obviously, we have

Lemma 5.1. The correspondence

$$
\varepsilon: H^{n}\left(Q^{n}\right) \rightarrow \prod_{j, t} H^{n}\left(P_{j}^{n}(t)\right)
$$

is an isomorphism. 
In the sequel, for convenience, the subcategories $F_{n}^{b}$ and $Q_{n}$ will be denoted by $K_{n}^{(4)}$ and $K_{n}^{(5)}$. Let $R=(R, *)$ be an arbitrary space from $K$. Let $q \in \Pi^{n}\left(R ; K_{n}^{(4)}\right)$ and

$$
\alpha=\left(Q^{n} ; f\right) \in{ }^{(5)} \omega(R ; n)
$$

be an arbitrary index. Assume $f_{j, t}=f i_{j, t}$ and consider the indices

$$
\alpha_{j, t}=\left(P^{n}(t) ; f_{j, t}\right) \in{ }^{(4)} \omega(R ; n) .
$$

Let

$$
p_{\alpha}=\left[\lambda_{4}(q)\right]_{\alpha}=\varepsilon^{-1}\left(\left\{q_{\alpha_{j, t}}\right\}\right) .
$$

We will show that the set $\left\{p_{\alpha}\right\}$ defines an element of the group $\Pi^{n}\left(R ; K_{n}^{(5)}\right)$ and the natural isomorphism

$$
\lambda_{4}: \Pi^{n}\left(R ; K_{n}^{(4)}\right) \rightarrow \Pi^{n}\left(R ; K_{n}^{(5)}\right) .
$$

By $\vee$ we will denote the symbol of finite one-point union of spaces. Consider the commutative diagram

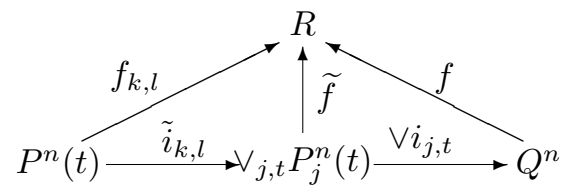

where $\widetilde{i}_{k, l}$ and $\vee i_{j, t}$ are standard embeddings, and the indices

$$
\begin{gathered}
\alpha=\left(Q^{n} ; f\right) \in{ }^{(5)} \omega(R ; n), \\
\beta=\left(\vee_{j, t} P_{j}^{n}(t) ; \widetilde{f}\right) \in{ }^{(4)} \omega(R ; n), \\
\alpha_{k, l}=\left(P^{n}(t) ; f_{k, l}\right) \in{ }^{(4)} \omega(R ; n) .
\end{gathered}
$$

We have $\left(\vee i_{j, t}\right) \widetilde{i}_{k, l}=i_{k, l}$. Therefore $\alpha_{k, l}<\beta$. Then

$$
\widetilde{i}_{k, l}^{*}\left(\left(\vee i_{j, t}\right)^{*}\left(p_{\alpha}\right)\right)=i_{k, l}^{*}\left(p_{\alpha}\right)=q_{\alpha_{k, l}} .
$$

Since this is true for arbitrary $k$ and $l$, we have

$$
\left(\vee i_{j, t}\right)^{*}\left(p_{\alpha}\right)=q_{\beta} .
$$

Now consider the diagram 


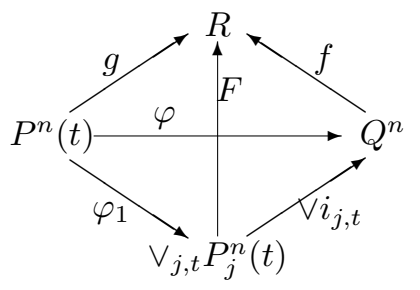

where $f \varphi \sim g, F=f\left(\vee i_{j, t}\right)$, and $\left(\vee i_{j, t}\right) \varphi_{1}=\varphi$ (since $P^{n}(t)$ is a compact space and $\varphi$ is a continuous map, it follows that there exists a map $\varphi_{1}$ ). Then

$$
F \varphi_{1}=f\left(\vee_{j, t} i_{j, t}\right) \varphi_{1}=f \varphi \sim g .
$$

Consider the indices

$$
\begin{gathered}
\beta=\left(P^{n}(t), g\right) \in{ }^{(4)} \omega(R ; n), \\
\beta_{1}=\left(\vee_{j, t} P_{j}^{n}(t) ; F\right) \in{ }^{(4)} \omega(R ; n) .
\end{gathered}
$$

Then $\beta<\beta_{1}$, and by (5) we have

$$
\varphi^{*}\left(p_{\alpha}\right)=\varphi_{1}^{*}\left(\left(\vee_{j, t}\right)^{*}\left(p_{\alpha}\right)\right)=\varphi_{1}^{*}\left(q_{\beta_{1}}\right)=q_{\beta} .
$$

Thus

$$
\varphi^{*}\left(p_{\alpha}\right)=q_{\beta} .
$$

Finally, consider the diagram

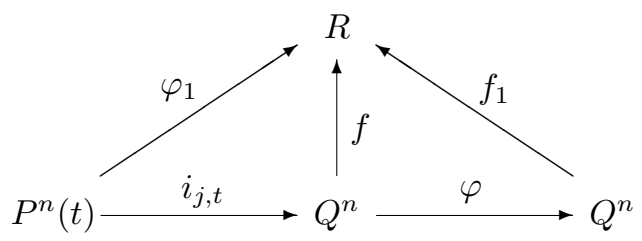

where $f_{1} \varphi \sim f$ and $f i_{j, t}=\varphi_{1}$. Consider the indices

$$
\begin{gathered}
\alpha_{1}=\left(Q^{n} ; f_{1}\right) \in{ }^{(5)} \omega(R ; n), \\
\alpha_{j, t}=\left(P^{n}(t) ; \varphi_{1}\right) \in{ }^{(4)} \omega(R ; n) .
\end{gathered}
$$

Thus $\alpha<\alpha_{1}$. Therefore by (6) we have

$$
i_{j, t}^{*}\left(\varphi^{*}\left(p_{\alpha_{1}}\right)\right)=\left(\varphi i_{j, t}\right)^{*}\left(p_{\alpha_{1}}\right)=q_{\alpha_{j, t}} .
$$

Since this equality is true for arbitrary $j$ and $t$, we obtain $\varphi^{*}\left(p_{\alpha_{1}}\right)=p_{\alpha}$. Therefore the set $\left\{p_{\alpha}\right\}$ defines an element $p \in \Pi^{n}\left(R ; K_{n}^{(5)}\right)$. The map $\lambda_{4}$ is now defined by setting $\lambda_{4}(q)=p$, where $q \in \Pi^{n}\left(R ; K_{n}^{(4)}\right)$. 
Let $q_{1}, q_{2} \in \Pi^{n}\left(R ; K_{n}^{(4)}\right)$. Then we have

$$
\begin{aligned}
& i_{j, t}^{*}\left(\left[\lambda_{4}\left(q_{1}+q_{2}\right)\right]_{\alpha}\right)=\left(q_{1}+q_{2}\right)_{\alpha_{j, t}}=\left(q_{1}\right)_{\alpha_{j, t}}+\left(q_{2}\right)_{\alpha_{j, t}}=i_{j, t}^{*}\left(\left[\lambda_{4}\left(q_{1}\right)\right]_{\alpha}\right)+ \\
& +i_{j, t}^{*}\left(\left[\lambda_{4}\left(q_{2}\right)\right]_{\alpha}\right)=i_{j, t}^{*}\left(\left[\lambda_{4}\left(q_{1}\right)\right]_{\alpha}+\left[\lambda_{4}\left(q_{2}\right)\right]_{\alpha}\right)=i_{j, t}^{*}\left(\left[\lambda_{4}\left(q_{1}\right)+\lambda_{4}\left(q_{2}\right)\right]_{\alpha}\right) .
\end{aligned}
$$

Since this equality holds for arbitrary $j$ and $t$, we have

$$
\lambda_{4}\left(q_{1}+q_{2}\right)=\lambda_{4}\left(q_{1}\right)+\lambda_{4}\left(q_{2}\right) .
$$

Let now $\varphi: S \rightarrow R$ be an arbitrary map. Consider the diagram

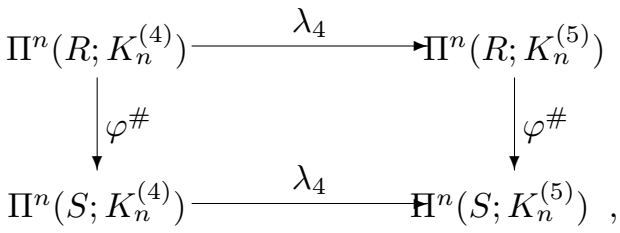

the element $q \in \Pi^{n}\left(R ; K_{n}^{(4)}\right)$, indices $\alpha, \alpha_{j, t}$, and

$$
\begin{gathered}
\beta=\varphi(\alpha)=\left(Q^{n} ; g\right) \in{ }^{(5)} \omega(R ; n), \\
\beta_{j, t}=\left(P^{n}(t) ; g_{j, t}\right) \in{ }^{(4)} \omega(R ; n),
\end{gathered}
$$

where $g=\varphi f, g_{j, t}=g i_{j, t}$. Then $\beta_{j, t}=\varphi\left(\alpha_{j, t}\right)$, and we have

$$
\left[\varphi^{\#}\left(\lambda_{4}(q)\right)\right]_{\alpha}=\left[\lambda_{4}(q)\right]_{\beta}=\varepsilon^{-1}\left(\left\{q_{\beta_{j, t}}\right\}\right) .
$$

On the other hand, we have

$$
\left[\lambda_{4}\left(\varphi^{\#}(q)\right)\right]_{\alpha}=\varepsilon^{-1}\left(\left\{\left[\varphi^{\#}(q)\right]_{\alpha_{j, t}}\right\}\right)=\varepsilon^{-1}\left(\left\{q_{\varphi\left(\alpha_{j, t}\right)}\right\}\right)=\varepsilon^{-1}\left(\left\{q_{\beta_{j, t}}\right\}\right) .
$$

Thus $\lambda_{4}$ is a natural homomorphism.

We will prove that $\lambda_{4}$ is a monomorphism. Let $q \in \Pi^{n}\left(R ; K_{n}^{(4)}\right)$ and $\lambda_{4}(q)=0$. Consider an arbitrary index

$$
\beta=\left(\vee_{j, t} P_{j}^{n}(t) ; g\right) \in{ }^{(4)} \omega(R ; n)
$$

and define the map $f: Q^{n} \rightarrow R$ by taking

$$
\begin{gathered}
f\left(\left(\vee i_{j, t}\right)(x)\right)=g(x), \quad x \in \vee_{j, t} P_{j}^{n}(t), \\
f\left(Q^{n}-\left(\vee i_{j, t}\right)\left(\vee_{j, t} P_{j}^{n}(t)\right)\right)=* .
\end{gathered}
$$

Consider the index

$$
\alpha=\left(Q^{n} ; f\right) \in{ }^{(5)} \omega(R ; n)
$$

and the commutative diagram 


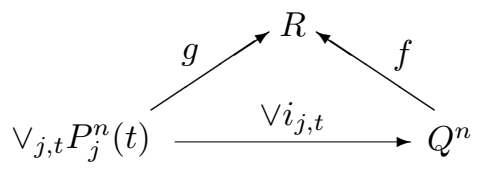

then by (5) we have

$$
q_{\beta}=\left(\vee_{j, t} i_{j, t}\right)^{*}\left(\left[\lambda_{4}(q)\right]_{\alpha}\right)=\left(\vee_{j, t} i_{j, t}\right)^{*}(0)=0 .
$$

Therefore $q=0$ and $\lambda_{4}$ is a monomorphism.

Further, we will prove that $\lambda_{4}$ is an epimorphism. Let $p \in \Pi^{n}\left(R ; K_{n}^{(5)}\right)$ and

$$
\alpha=(X ; f) \in{ }^{(4)} \omega(R ; n)
$$

be an arbitrary index, $X \in K_{n}^{(4)}$. Therefore the space $X$ can be represented in the form $X=\vee_{j, t} P_{j}^{n}(t)$, where $j$ is an index indicating a certain arrangement of the identical subspaces of $X$. Then we have the natural embedding $i: X \rightarrow Q^{n}$. Define the map $l: Q^{n} \rightarrow X$ by taking

$$
\begin{aligned}
& l(i(x))=x, \quad x \in X ; \\
& l\left(Q^{n}-i(X)\right)=* .
\end{aligned}
$$

Let $g=f l$. We have $g i=f$. Let

$$
\beta=\left(Q^{n} ; g\right) \in{ }^{(5)} \omega(R ; n) .
$$

Assume that

$$
q_{\alpha}=i^{*}\left(p_{\beta}\right) .
$$

Consider a different arrangement of subspaces of $X$. Let the map $\widetilde{i}$ and the index $\widetilde{\beta}=\left(Q^{n} ; \widetilde{g}\right)$ be defined in the same way as $i$ and $\beta$, respectively. Consider the commutative diagram

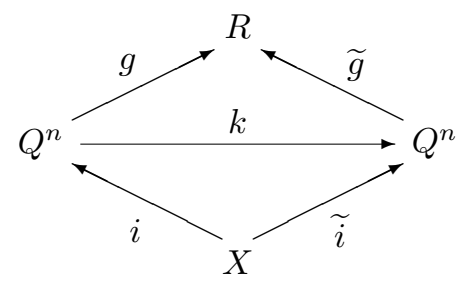

where the map $k$ can be defined by a certain permutation of subspaces of $Q^{n}$. Then $\beta<\widetilde{\beta}$ and we have

$$
\widetilde{i}^{*}\left(p_{\widetilde{\beta}}\right)=i^{*}\left(k^{*}\left(p_{\widetilde{\beta}}\right)\right)=i^{*}\left(p_{\beta}\right) .
$$

Thus definition (7) is correct.

Consider the diagram 


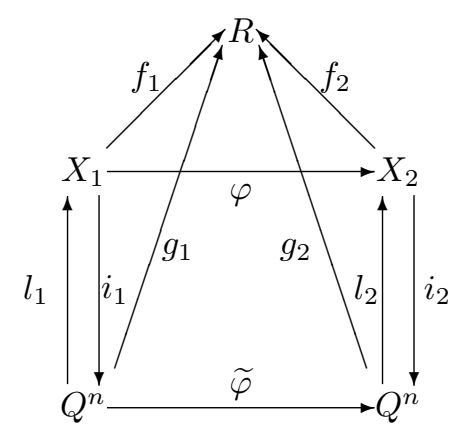

where $X_{1}, X_{2} \in K_{n}^{(4)}, f_{2} \varphi \sim f_{1}, \widetilde{\varphi}=i_{2} \varphi l_{1}, f_{1}=g_{1} i_{1}, f_{2}=g_{2} i_{2}$. Then

$$
\begin{gathered}
\widetilde{\varphi} i_{1}=i_{2} \varphi l_{1} i_{1}=i_{2} \varphi, \\
g_{2} \widetilde{\varphi}=f_{2} l_{2} i_{2} \varphi l_{1}=f_{2} \varphi l_{1} \sim f_{1} l_{1}=g_{1} .
\end{gathered}
$$

Consider the indices

$$
\begin{array}{ll}
\alpha_{t} & =\left(X_{t} ; f_{t}\right) \in{ }^{(4)} \omega(R ; n), \quad t=1,2 ; \\
\beta_{t}=\left(Q^{n} ; g_{t}\right) \in{ }^{(5)} \omega(R ; n), & t=1,2 .
\end{array}
$$

Then $\alpha_{1}<\alpha_{2}, \beta_{1}<\beta_{2}$ and we have

$$
\varphi^{*}\left(q_{\alpha_{2}}\right)=\varphi^{*}\left(i_{2}^{*}\left(p_{\beta_{2}}\right)\right)=i_{1}^{*}\left(\widetilde{\varphi}^{*}\left(p_{\beta_{2}}\right)\right)=i_{1}^{*}\left(p_{\beta_{1}}\right)=q_{\alpha_{1}} .
$$

Therefore the set $\left\{q_{\alpha}\right\}$ defines an element $q \in \Pi^{n}\left(R ; K_{n}^{(4)}\right)$.

Finally, let us prove that $\lambda_{4}(q)=p$. Consider an arbitrary index

$$
\alpha=\left(Q^{n} ; f\right) \in{ }^{(5)} \omega(R ; n)
$$

and the index

$$
\alpha_{j, t}=\left(P^{n}(t) ; f i_{j, t}\right) \in{ }^{(4)} \omega(R ; n) .
$$

Then $i_{j, t}=i$ and we have

$$
i_{j, t}^{*}\left(\left[\lambda_{4}(q)\right]_{\alpha}\right)=q_{\alpha_{j, t}}=i_{j, t}^{*}\left(p_{\alpha}\right) .
$$

Since this equality is true for arbitrary $j$ and $t$, we have $\left[\lambda_{4}(q)\right]_{\alpha}=p_{\alpha}$. Therefore $\lambda_{4}(q)=p$. This completes the proof of Theorem 2.5.

\section{REFERENCES}

1. F.W. Bauer, Homotopie und homologie. Math. Ann. 149(1963), 105-130.

2. G.S. Chogoshvili, On functors generated by cohomology. (Russian) Bull. Acad. Sci. Georgian SSR 97(1980), No. 2, 273-276. 
3. G.S. Chogoshvili, On the relation of the $D$-functor to analogous functors. (Russian) Bull. Acad. Sci. Georgian SSR 108(1982), No. 3, 473-476.

4. W. Hurewicz, I. Dugundji, and C.H. Dowker, Continuous connectivity groups in terms of limit groups. Ann. Math. 49(1948), 391-406.

5. S.M. Khazhomia, On some properties of functors dual to homotopy functors. (Russian) Trudy Tbiliss. Mat. Inst. Razmadze 91(1988), 81-97.

6. —- On the dual homotopy functors. Bull. Acad. Sci. Georgia 146(1992), No. 1, 13-16.

7. —- On Chogoshvili cohomotopies. Bull. Acad. Sci. Georgia (to appear).

(Received 11.09.1992)

Author's address:

A.Razmadze Mathematical Institute

Georgian Academy of Sciences

1, Z. Rukhadze St., 380093 Tbilisi

Republic of Georgia 